(C) 2015 IEEE. Personal use of this material is permitted. Permission from IEEE must be obtained for all other uses, in any current or future media, including reprinting/republishing this material for advertising or promotional purposes, creating new collective works, for resale or redistribution to servers or lists, or reuse of any copyrighted component of this work in other works 


\title{
Will the Area Spectral Efficiency Monotonically Grow as Small Cells Go Dense?
}

\author{
Ming Ding, National ICT Australia (NICTA), Australia \{Ming.Ding@nicta.com.au\} \\ David López-Pérez, Bell Labs Alcatel-Lucent, Ireland \{dr.david. lopez@ieee.org\} \\ Guoqiang Mao, The University of Technology Sydney and NICTA, Australia \{g.mao@ieee.org\} \\ Peng Wang, The University of Sydney and NICTA, Australia \{thomaspeng.wang@sydney.edu.au \\ Zihuai Lin, The University of Sydney, Australia \{zihuai.lin@sydney.edu.au\}
}

\begin{abstract}
In this paper, we introduce a sophisticated path loss model into the stochastic geometry analysis incorporating both line-of-sight (LoS) and non-line-of-sight (NLoS) transmissions to study their performance impact in small cell networks (SCNs). Analytical results are obtained on the coverage probability and the area spectral efficiency (ASE) assuming both a general path loss model and a special case of path loss model recommended by the 3rd Generation Partnership Project (3GPP) standards. The performance impact of LoS and NLoS transmissions in SCNs in terms of the coverage probability and the ASE is shown to be significant both quantitatively and qualitatively, compared with previous work that does not differentiate LoS and NLoS transmissions. Particularly, our analysis demonstrates that when the density of small cells is larger than a threshold, the network coverage probability will decrease as small cells become denser, which in turn makes the ASE suffer from a slow growth or even a notable decrease. For practical regime of small cell density, the performance results derived from our analysis are distinctively different from previous results, and shed new insights on the design and deployment of future dense/ultra-dense SCNs.
\end{abstract}

\section{INTRODUCTION}

Dense small cell networks ( $\mathrm{SCNs}$ ) are considered as the most promising approach to rapidly increase network capacity and meet the ever-increasing capacity demands in the 5th generation (5G) systems [1]. However, up to now, most theoretical studies on SCNs only consider simple path loss models that do not differentiate Line-of-Sight (LoS) and Non-Line-of-Sight (NLoS) transmissions [2-5]. The major conclusion [2-5] is that neither the number of small cells nor the number of cell tiers changes the coverage probability in interference-limited fully-loaded cellular networks. Such conclusion implies that the area spectral efficiency (ASE) will monotonically grow as small cells go dense. An intriguing question is: Does this optimistic conclusion still hold when practical LoS and NLoS transmissions are considered in SCNs?

It is well-known that LoS transmission often occurs when the distance between a transmitter and a receiver is small, while NLoS is more common in long-distance transmissions as well as in office environments and in central business districts. For a given network environment, when the distance between transmitter and receiver decreases, the probability that an LoS path exists between them increases, causing a transition from NLoS transmission to LoS transmission.

To the best of authors' knowledge, up to now, performance analysis considering both LoS and NLoS transmissions are [6] and [7]. In [6], the authors assumed a multi-slope piecewise path loss function. Such multi-slope piece-wise path loss function does not fit well with the NLoS and LoS model defined by the 3rd Generation Partnership Project (3GPP) standards, in which the path loss function is not a one-toone mapping to the distance [8]. In [7], the authors treated the event of LoS or NLoS transmission as a probabilistic event for a millimetre wave communication scenario. To simplify the analysis, the LoS probability function was approximated by a moment-matched equivalent step function. The single-piece path loss model and the proposed step function for modeling the transition from NLoS to LoS transmissions are also not compatible with the model recommended by the 3GPP $[8,9]$.

In this paper, we use a general path loss model that features piece-wise path loss functions with probabilistic LoS and NLoS transmissions. Note that the proposed model is very general and includes almost all existing models used to capture LoS and NLoS transmissions [6-9] as its special cases. The main contributions of the paper are as follows:

- Analytical results are obtained for the coverage probability and the ASE using a general path loss model incorporating both LoS and NLoS transmissions.

- Using the above results, closed-form expressions are further obtained for the coverage probability and the ASE for a special case based on the 3GPP standards.

- Our theoretical analysis reveals an important finding, i.e., the ASE will initially increase with the increase of the small cell density, but when the density of small cells becomes sufficiently large, the network coverage probability will decrease as small cells become denser. This in turn makes the ASE suffer from a slow growth or even a notable decrease. Thereafter, when the small cell density is very large, the ASE will then grow almost linearly with the network densification. These results are not only quantitatively but also qualitatively different from previous study results [2-7]. Thus, our results shed new insights on the design and deployment of future dense/ultra-dense SCNs in realistic environments.

The remainder of this paper is structured as follows. Section II describes the system model. Section III presents 
our main analytical results on the coverage probability and the ASE, followed by their application in a 3GPP case in Section IV. The derived results are validated using simulations in Section V. Finally, the conclusions are drawn in Section VI.

\section{SYSTEM MODEL}

We consider a DL cellular network in which BSs are deployed in a plane according to a homogeneous Poisson point process (HPPP) $\Phi$ of intensity $\lambda \mathrm{BSs} / \mathrm{km}^{2}$. UEs are Poisson distributed in the considered network with an intensity of $\lambda^{\mathrm{UE}}$ $\mathrm{BSs} / \mathrm{km}^{2} . \lambda^{\mathrm{UE}}$ is assumed to be sufficiently larger than $\lambda$ so that each BS has at least one associated UE in its coverage area. The distance between an arbitrary BS and an arbitrary UE is denoted by $r$ in $\mathrm{km}$. Considering practical LoS and NLoS transmissions, we propose to model the path loss associated with distance $r$ as (1), shown on the top of the next page.

In (1), the path loss function $\zeta(r)$ is segmented into $N$ pieces with the $N$-th piece $\zeta_{n}(r), n \in\{1,2, \ldots, N\}$. For each $\zeta_{n}(r), \zeta_{n}^{\mathrm{L}}(r)$ is the $n$-th piece of the path loss function for LoS transmission, $\zeta_{n}^{\mathrm{NL}}(r)$ is the $n$-th piece of the path loss function for NLoS transmission and $\operatorname{Pr}_{n}^{\mathrm{L}}(r)$ is the $n$-th piece of the LoS probability function. In more detail,

- $\zeta_{n}(r)$ is modeled as

$$
\zeta_{n}(r)=\left\{\begin{array}{ll}
\zeta_{n}^{\mathrm{L}}(r)=A_{n}^{\mathrm{L}} r^{-\alpha_{n}^{\mathrm{L}},} & \text { for } \mathrm{LoS} \\
\zeta_{n}^{\mathrm{NL}}(r)=A_{n}^{\mathrm{NL}} r^{-\alpha_{n}^{\mathrm{NL}}}, & \text { for NLoS }
\end{array},\right.
$$

with $A_{n}^{\mathrm{L}}$ and $A_{n}^{\mathrm{NL}}, n \in\{1,2, \ldots, N\}$ being the path losses at a reference distance $r=1$ and $\alpha_{n}^{\mathrm{L}}$ and $\alpha_{n}^{\mathrm{NL}}, n \in\{1,2, \ldots, N\}$ being the path loss exponents for the LoS and the NLoS cases in $\zeta_{n}(r)$, respectively. In practice, $A_{n}^{\mathrm{L}}, A_{n}^{\mathrm{NL}}, \alpha_{n}^{\mathrm{L}}$ and $\alpha_{n}^{\mathrm{NL}}$ are constants obtained from field tests $[8,9]$.

- $\operatorname{Pr}_{n}^{\mathrm{L}}(r)$ is the $n$-th piece probability function that a transmitter and a receiver separated by a distance $r \in$ $\left[d_{n-1}, d_{n}\right)$ has an LoS path, which is usually a monotonically decreasing function of $r$. For convenience, $\left\{\operatorname{Pr}_{n}^{\mathrm{L}}(r)\right\}$ is further stacked into a piece-wise LoS probability function as

$$
\operatorname{Pr}^{\mathrm{L}}(r)=\left\{\begin{array}{ll}
\operatorname{Pr}_{1}^{\mathrm{L}}(r), & \text { when } 0 \leq r \leq d_{1} \\
\operatorname{Pr}_{2}^{\mathrm{L}}(r), & \text { when } d_{1}<r \leq d_{2} \\
\vdots & \vdots \\
\operatorname{Pr}_{N}^{\mathrm{L}}(r), & \text { when } r>d_{N-1}
\end{array} .\right.
$$

Our model is consistent with the ones adopted in the 3 GPP $[8,9]$. Note that the considered path loss model in (1) will degenerate to that adopted in [6] and [7] when $\operatorname{Pr}_{n}^{\mathrm{L}}(r)=$ $0, \forall n \in\{1,2, \ldots, N\}$ and $N=1$, respectively.

As a common practice in the field [2-6], each UE is assumed to be associated with the nearest BS to the UE, and the multipath fading between an arbitrary BS and an arbitrary UE is modeled as independently identical distributed (i.i.d.) Rayleigh fading, i.e., the channel gain is denoted by $h$ and is modeled as an i.i.d. exponential random variable (RV). The transmit power of each BS and the additive white Gaussian noise (AWGN) power at each UE are denoted by $P$ and $N_{0}$, respectively.

\section{Analysis Based on General Path Loss Model}

Using the properties of the HPPP, we study the performance of $\mathrm{SCNs}$ by considering the performance of a typical UE located at the origin $o$. We first investigate the coverage probability and thereafter the ASE.

The coverage probability is defined as the probability that the signal to interference plus noise ratio (SINR) of the typical UE, denoted by SINR, is above a threshold $\gamma$ :

$$
p^{\operatorname{cov}}(\lambda, \gamma)=\operatorname{Pr}[\operatorname{SINR}>\gamma]
$$

where the SINR is computed by

$$
\mathrm{SINR}=\frac{P \zeta(r) h}{I_{r}+N_{0}},
$$

where $I_{r}$ is the cumulative interference given by

$$
I_{r}=\sum_{i \in \Phi \backslash b_{o}} P \beta_{i} g_{i},
$$

where $b_{o}$ is the BS associated with the typical UE and located at distance $r$ from the typical UE, and $\beta_{i}$ and $g_{i}$ are the path loss and the multi-path fading channel gain associated with the $i$-th interfering BS, respectively.

According to [6] and [7], the area spectral efficiency (ASE) in $\mathrm{bps} / \mathrm{Hz} / \mathrm{km}^{2}$ for a given $\lambda$ can be computed by

$$
A^{\mathrm{ASE}}\left(\lambda, \gamma_{0}\right)=\lambda \int_{\gamma_{0}}^{\infty} \log _{2}(1+x) f_{X}(\lambda, x) d x,
$$

where $\gamma_{0}$ is the minimum working SINR for the considered $\mathrm{SCN}$, and $f_{X}(\lambda, x)$ is the probability density function (PDF) of SINR observed at the typical UE at a particular value of $\lambda$.

Since $p^{\text {cov }}(\lambda, \gamma)$ can be defined as the complementary cumulative distribution function (CCDF) of SINR, $f_{X}(\lambda, x)$ can be expressed as

$$
f_{X}(\lambda, x)=\frac{\partial\left(1-p^{\operatorname{cov}}(\lambda, x)\right)}{\partial x} .
$$

Given the definition of the coverage probability and the ASE presented in (4) and (7) respectively, and using the path loss model of (1), we present our main result on $p^{\text {cov }}(\lambda, \gamma)$ in Theorem 1 shown on the next page. Plugging $p^{\operatorname{cov}}(\lambda, \gamma)$ from (9) of Theorem 1 into (8), we can get the ASE using (7).

As can be seen from Theorem 1, the coverage probability, $p^{\text {cov }}(\lambda, \gamma)$, is a function of the piece-wise path loss function $\left\{\zeta_{n}(r)\right\}$ and the piece-wise LoS probability function $\left\{\operatorname{Pr}_{n}^{\mathrm{L}}(r)\right\}$. We will investigate their impacts in the sequel.

\section{Study of a 3GPP Special Case}

As a special case of Theorem 1, we consider the path loss function, $\zeta(r)$,

$$
\zeta(r)= \begin{cases}A^{\mathrm{L}} r^{-\alpha^{\mathrm{L}}}, & \text { w/ probability } \operatorname{Pr}^{\mathrm{L}}(r) \\ A^{\mathrm{NL}} r^{-\alpha^{\mathrm{NL}}}, & \text { w/ probability }\left(1-\operatorname{Pr}^{\mathrm{L}}(r)\right)\end{cases}
$$

together with the linear LoS probability function, which is

$$
\operatorname{Pr}^{\mathrm{L}}(r)=\left\{\begin{array}{ll}
1-\frac{r}{d_{1}}, & 0<r \leq d_{1} \\
0, & r>d_{1}
\end{array},\right.
$$

both respectively recommended in the 3GPP [8, 9].

Considering the general path loss model presented in (1), the path loss model presented in (14) and (15) can be deemed 


$$
\zeta(r)=\left\{\begin{array}{ll}
\zeta_{1}(r)=\left\{\begin{array}{ll}
\zeta_{1}^{\mathrm{L}}(r), & \text { with probability } \operatorname{Pr}_{1}^{\mathrm{L}}(r) \\
\zeta_{1}^{\mathrm{NL}}(r), & \text { with probability }\left(1-\operatorname{Pr}_{1}^{\mathrm{L}}(r)\right) \\
\zeta_{2}^{\mathrm{L}}(r), & \text { with probability } \operatorname{Pr}_{2}^{\mathrm{L}}(r) \\
\zeta_{2}^{\mathrm{NL}}(r), & \text { with probability }\left(1-\operatorname{Pr}_{2}^{\mathrm{L}}(r)\right)
\end{array}, \text { when } 0 \leq r \leq d_{1}\right. \\
\vdots \\
\zeta_{N}(r)=\left\{\begin{array}{ll}
\zeta_{N}^{\mathrm{L}}(r), & \text { with probability } \operatorname{Pr}_{N}^{\mathrm{L}}(r) \\
\zeta_{N}^{\mathrm{NL}}(r), & \text { with probability }\left(1-\operatorname{Pr}_{N}^{\mathrm{L}}(r)\right)
\end{array}, \text { when } r>d_{N-1}\right.
\end{array} .\right.
$$

Theorem 1. Considering the path loss model of $(1), p^{\text {cov }}(\lambda, \gamma)$ can be derived as

$$
p^{\text {cov }}(\lambda, \gamma)=\sum_{n=1}^{N}\left(T_{n}^{L}+T_{n}^{N L}\right),
$$

where $T_{n}^{L}=\int_{d_{n-1}}^{d_{n}} \operatorname{Pr}\left[\frac{P \zeta_{n}^{L}(r) h}{I_{r}+N_{0}}>\gamma\right] f_{R, n}^{L}(r) d r, T_{n}^{N L}=\int_{d_{n-1}}^{d_{n}} \operatorname{Pr}\left[\frac{P \zeta_{n}^{N L}(r) h}{I_{r}+N_{0}}>\gamma\right] f_{R, n}^{N L}(r) d r$, and do and d $d_{N}$ are respectively defined as 0 and $\infty$. Moreover, $f_{R, n}^{L}(r)$ and $f_{R, n}^{N L}(r)$ are represented as

$$
f_{R, n}^{L}(r)=P r_{n}^{L}(r) \times \exp \left(-\pi r^{2} \lambda\right) \times 2 \pi r \lambda, \quad\left(d_{n-1}<r \leq d_{n}\right),
$$

and

$$
f_{R, n}^{N L}(r)=\left(1-\operatorname{Pr}_{n}^{L}(r)\right) \times \exp \left(-\pi r^{2} \lambda\right) \times 2 \pi r \lambda, \quad\left(d_{n-1}<r \leq d_{n}\right) .
$$

Furthermore, $\operatorname{Pr}\left[\frac{P \zeta_{n}^{L}(r) h}{I_{r}+N_{0}}>\gamma\right]$ and $\operatorname{Pr}\left[\frac{P \zeta_{n}^{N L}(r) h}{I_{r}+N_{0}}>\gamma\right]$ are respectively computed by

$$
\operatorname{Pr}\left[\frac{P \zeta_{n}^{L}(r) h}{I_{r}+N_{0}}>\gamma\right]=\exp \left(-\frac{\gamma N_{0}}{P \zeta_{n}^{L}(r)}\right) \mathscr{L}_{I_{r}}\left(\frac{\gamma}{P \zeta_{n}^{L}(r)}\right)
$$

and

$$
\operatorname{Pr}\left[\frac{P \zeta_{n}^{N L}(r) h}{I_{r}+N_{0}}>\gamma\right]=\exp \left(-\frac{\gamma N_{0}}{P \zeta_{n}^{N L}(r)}\right) \mathscr{L}_{I_{r}}\left(\frac{\gamma}{P \zeta_{n}^{N L}(r)}\right),
$$

where $\mathscr{L}_{I_{r}}(s)$ is the Laplace transform of $R V I_{r}$ evaluated at $s$.

Proof: See Appendix A.

as a special case of (1) with the following substitution: $N=2$, $\zeta_{1}^{\mathrm{L}}(r)=\zeta_{2}^{\mathrm{L}}(r)=A^{\mathrm{L}} r^{-\alpha^{\mathrm{L}}}, \zeta_{1}^{\mathrm{NL}}(r)=\zeta_{2}^{\mathrm{NL}}(r)=A^{\mathrm{NL}} r^{-\alpha^{\mathrm{NL}}}$, $\operatorname{Pr}_{1}^{\mathrm{L}}(r)=1-\frac{r}{d_{1}}$, and $\operatorname{Pr}_{2}^{\mathrm{L}}(r)=0$. For clarity, this $3 \mathrm{GPP}$ special case is referred to as 3GPP Case 1 in the sequel.

According to Theorem $1, p^{\text {cov }}(\lambda, \gamma)$ can be obtained as

$$
p^{\mathrm{cov}}(\lambda, \gamma)=\sum_{n=1}^{2}\left(T_{n}^{\mathrm{L}}+T_{n}^{\mathrm{NL}}\right) .
$$

In the following subsections, we investigate $T_{1}^{\mathrm{L}}, T_{1}^{\mathrm{NL}}, T_{2}^{\mathrm{L}}$, and $T_{2}^{\mathrm{NL}}$, respectively.

\section{A. The Computation of $T_{1}^{L}$ for $3 G P P$ Case 1}

From Theorem 1, $T_{1}^{\mathrm{L}}$ can be derived as

$T_{1}^{\mathrm{L}}=\int_{0}^{d_{1}} \exp \left(-\frac{\gamma r^{\alpha^{\mathrm{L}}} N_{0}}{P A^{\mathrm{L}}}\right) \mathscr{L}_{I_{r}}\left(\frac{\gamma r^{\alpha^{\mathrm{L}}}}{P A^{\mathrm{L}}}\right) f_{R, 1}^{\mathrm{L}}(r) d r$,

where according to Theorem 1 and (15), $f_{R, 1}^{\mathrm{L}}(r)$ becomes $f_{R, 1}^{\mathrm{L}}(r)=$

$$
\left(1-\frac{r}{d_{1}}\right) \times \exp \left(-\pi r^{2} \lambda\right) \times 2 \pi r \lambda, \quad\left(0<r \leq d_{1}\right) .
$$

Furthermore, to compute $\mathscr{L}_{I_{r}}\left(\frac{\gamma r^{\alpha^{\mathrm{L}}}}{P A^{\mathrm{L}}}\right)$ in the range of $0<$ $r \leq d_{1}$, we propose Lemma 2 .
Lemma 2. $\mathscr{L}_{I_{r}}\left(\frac{\gamma r^{\alpha^{\mathrm{L}}}}{P A^{\mathrm{L}}}\right)$ in the range of $0<r \leq d_{1}$ can be calculated by

$$
\begin{aligned}
& \mathscr{L}_{I_{r}}\left(\frac{\gamma r^{\alpha^{\mathrm{L}}}}{P A^{\mathrm{L}}}\right)= \\
& \exp \left(-2 \pi \lambda\left(\rho_{1}\left(\alpha^{\mathrm{L}}, 1,\left(\gamma r^{\alpha^{\mathrm{L}}}\right)^{-1}, d_{1}\right)\right.\right. \\
& \left.\left.-\rho_{1}\left(\alpha^{\mathrm{L}}, 1,\left(\gamma r^{\alpha^{\mathrm{L}}}\right)^{-1}, r\right)\right)\right) \\
& \times \exp \left(\frac { 2 \pi \lambda } { d _ { 0 } } \left(\rho_{1}\left(\alpha^{\mathrm{L}}, 2,\left(\gamma r^{\alpha^{\mathrm{L}}}\right)^{-1}, d_{1}\right)\right.\right. \\
& \left.\left.-\rho_{1}\left(\alpha^{\mathrm{L}}, 2,\left(\gamma r^{\alpha^{\mathrm{L}}}\right)^{-1}, r\right)\right)\right) \\
& \times \exp \left(-\frac{2 \pi \lambda}{d_{0}}\left(\rho_{1}\left(\alpha^{\mathrm{NL}}, 2,\left(\frac{\gamma A^{\mathrm{NL}}}{A^{\mathrm{L}}} r^{\alpha^{\mathrm{L}}}\right)^{-1}, d_{1}\right)\right.\right. \\
& \left.\left.-\rho_{1}\left(\alpha^{\mathrm{NL}}, 2,\left(\frac{\gamma A^{\mathrm{NL}}}{A^{\mathrm{L}}} r^{\alpha^{\mathrm{L}}}\right)^{-1}, r\right)\right)\right) \\
& \times \exp \left(-2 \pi \lambda \rho_{2}\left(\alpha^{\mathrm{NL}}, 1,\left(\frac{\gamma A^{\mathrm{NL}}}{A^{\mathrm{L}}} r^{\alpha^{\mathrm{L}}}\right)^{-1}, d_{1}\right)\right),
\end{aligned}
$$

where $\left(0<r \leq d_{1}\right)$, 
$\rho_{1}(\alpha, \beta, t, d)=$

and $\left[\frac{d^{(\beta+1)}}{\beta+1}\right]{ }_{2} F_{1}\left[1, \frac{\beta+1}{\alpha} ; 1+\frac{\beta+1}{\alpha} ;-t d^{\alpha}\right]$,

$\rho_{2}(\alpha, \beta, t, d)=$

$\left[\frac{d^{-(\alpha-\beta-1)}}{t(\alpha-\beta-1)}\right]{ }_{2} F_{1}\left[1,1-\frac{\beta+1}{\alpha} ; 2-\frac{\beta+1}{\alpha} ;-\frac{1}{t d^{\alpha}}\right]$,

where ${ }_{2} F_{1}[\cdot, \cdot ; \cdot ; \cdot]$ is the hyper-geometric function [10].

Proof: See Appendix B.

\section{B. The Computation of $T_{1}^{N L}$ for $3 G P P$ Case 1}

From Theorem 1, $T_{1}^{\mathrm{NL}}$ can be derived as

$T_{1}^{\mathrm{NL}}=\int_{0}^{d_{1}} \exp \left(-\frac{\gamma r^{\alpha^{\mathrm{NL}}} N_{0}}{P A^{\mathrm{NL}}}\right) \mathscr{L}_{I_{r}}\left(\frac{\gamma r^{\alpha^{\mathrm{NL}}}}{P A^{\mathrm{NL}}}\right) f_{R, 1}^{\mathrm{NL}}(r) d r$,

where according to Theorem 1 and (15), $f_{R, 1}^{\mathrm{NL}}(r)$ becomes

$f_{R, 1}^{\mathrm{NL}}(r)=\frac{r}{d_{1}} \times \exp \left(-\pi r^{2} \lambda\right) \times 2 \pi r \lambda, \quad\left(0<r \leq d_{1}\right)$.

Furthermore, to compute $\mathscr{L}_{I_{r}}\left(\frac{\gamma r^{\alpha^{\mathrm{NL}}}}{P A^{\mathrm{NL}}}\right)$ in the range of $0<$ $r \leq d_{1}$, we propose Lemma 3 .

Lemma 3. $\mathscr{L}_{I_{r}}\left(\frac{\gamma r^{\alpha^{\mathrm{NL}}}}{P A^{\mathrm{NL}}}\right)$ in the range of $0<r \leq d_{1}$ can be calculated by

$$
\begin{array}{r}
\mathscr{L}_{I_{r}}\left(\frac{\gamma r^{\alpha^{\mathrm{NL}}}}{P A^{\mathrm{NL}}}\right)= \\
\exp \left(-2 \pi \lambda\left(\rho_{1}\left(\alpha^{\mathrm{L}}, 1,\left(\frac{\gamma A^{\mathrm{L}}}{A^{\mathrm{NL}}} r^{\alpha^{\mathrm{NL}}}\right)^{-1}, d_{1}\right)\right.\right. \\
\left.\left.\quad-\rho_{1}\left(\alpha^{\mathrm{L}}, 1,\left(\frac{\gamma A^{\mathrm{L}}}{A^{\mathrm{NL}}} r^{\alpha^{\mathrm{NL}}}\right)^{-1}, r\right)\right)\right) \\
\times \exp \left(\frac { 2 \pi \lambda } { d _ { 0 } } \left(\rho_{1}\left(\alpha^{\mathrm{L}}, 2,\left(\frac{\gamma A^{\mathrm{L}}}{A^{\mathrm{NL}}} r^{\alpha^{\mathrm{NL}}}\right)^{-1}, d_{1}\right)\right.\right. \\
\left.\left.\left.\quad-\rho_{1}\left(\alpha^{\mathrm{L}}, 2,\left(\frac{\gamma A^{\mathrm{L}}}{A^{\mathrm{NL}}} r^{\alpha^{\mathrm{NL}}}\right)^{-1}, r\right)\right)\right)\right) \\
\times \exp \left(-\frac{2 \pi \lambda}{d_{0}}\left(\rho_{1}\left(\alpha^{\mathrm{NL}}, 2,\left(\gamma r^{\alpha^{\mathrm{NL}}}\right)^{-1}, d_{1}\right)\right.\right. \\
\left.\left.\quad-\rho_{1}\left(\alpha^{\mathrm{NL}}, 2,\left(\gamma r^{\alpha^{\mathrm{NL}}}\right)^{-1}, r\right)\right)\right) \\
\times \exp \left(-2 \pi \lambda \rho_{2}\left(\alpha^{\mathrm{NL}}, 1,\left(\gamma r^{\alpha^{\mathrm{NL}}}\right)^{-1}, d_{1}\right)\right)
\end{array}
$$

where $\rho_{1}(\alpha, \beta, t, d)$ and $\rho_{2}(\alpha, \beta, t, d)$ are defined in (20) and (21), respectively.

Proof: See Appendix C.

\section{The Computation of $T_{2}^{L}$ for 3GPP Case 1}

From Theorem 1, $T_{2}^{\mathrm{L}}$ can be derived as

$$
T_{2}^{\mathrm{L}}=\int_{d_{1}}^{\infty} \exp \left(-\frac{\gamma r^{\alpha^{\mathrm{L}}} N_{0}}{P A^{\mathrm{L}}}\right) \mathscr{L}_{I_{r}}\left(\frac{\gamma r^{\alpha^{\mathrm{L}}}}{P A^{\mathrm{L}}}\right) f_{R, 2}^{\mathrm{L}}(r) d r
$$$$
=0 \text {. }
$$

Note that the reason why $T_{2}^{\mathrm{L}}=0$ in (25) is because according to Theorem 1 and (15), we have

$$
\begin{aligned}
f_{R, 2}^{\mathrm{L}}(r) & =0 \times \exp \left(-\pi r^{2} \lambda\right) \times 2 \pi r \lambda \\
& =0, \quad\left(r>d_{1}\right) .
\end{aligned}
$$

\section{The Computation of $T_{2}^{N L}$ for 3GPP Case 1}

From Theorem $1, T_{2}^{\mathrm{NL}}$ can be derived as

$T_{2}^{\mathrm{NL}}=\int_{d_{1}}^{\infty} \exp \left(-\frac{\gamma r^{\alpha^{\mathrm{NL}}} N_{0}}{P A^{\mathrm{NL}}}\right) \mathscr{L}_{I_{r}}\left(\frac{\gamma r^{\alpha^{\mathrm{NL}}}}{P A^{\mathrm{NL}}}\right) f_{R, 2}^{\mathrm{NL}}(r) d r$

where according to Theorem 1 and (15), $f_{R, 2}^{\mathrm{NL}}(r)$ becomes

$$
\begin{aligned}
f_{R, 2}^{\mathrm{NL}}(r) & =1 \times \exp \left(-\pi r^{2} \lambda\right) \times 2 \pi r \lambda \\
& =\exp \left(-\pi \lambda r^{2}\right) \times 2 \pi r \lambda, \quad\left(r>d_{1}\right) .
\end{aligned}
$$

Furthermore, to compute $\mathscr{L}_{I_{r}}\left(\frac{\gamma r^{\alpha^{\mathrm{NL}}}}{P A^{\mathrm{NL}}}\right)$ in the range of $r>$ $d_{1}$, we propose Lemma 4.

Lemma 4. $\mathscr{L}_{I_{r}}\left(\frac{\gamma r^{\alpha^{\mathrm{NL}}}}{P A^{\mathrm{NL}}}\right)$ in the range of $r>d_{1}$ can be calculated by

$$
\begin{aligned}
& \mathscr{L}_{I_{r}}\left(\frac{\gamma r^{\mathrm{NL}}}{P A^{\mathrm{NL}}}\right)= \\
& \quad \exp \left(-2 \pi \lambda \rho_{2}\left(\alpha^{\mathrm{NL}}, 1,\left(\gamma r^{\alpha^{\mathrm{NL}}}\right)^{-1}, r\right)\right),\left(r>d_{1}\right),
\end{aligned}
$$

where $\rho_{2}(\alpha, \beta, t, d)$ is defined in (21).

\section{Proof: See Appendix D.}

E. The Results of $p^{\text {cov }}(\lambda, \gamma)$ and $A^{A S E}\left(\lambda, \gamma_{0}\right)$

To sum up, $p^{\text {cov }}(\lambda, \gamma)$ for 3GPP Case 1 can be written as

$$
p^{\mathrm{cov}}(\lambda, \gamma)=T_{1}^{\mathrm{L}}+T_{1}^{\mathrm{NL}}+T_{2}^{\mathrm{NL}},
$$

where $T_{1}^{\mathrm{L}}, T_{1}^{\mathrm{NL}}$ and $T_{2}^{\mathrm{NL}}$ are computed from closed-form expressions using (17), (22) and (27), respectively.

Plugging $p^{\operatorname{cov}}(\lambda, \gamma)$ obtained from (30) into (8), we can get the result of $A^{\mathrm{ASE}}\left(\lambda, \gamma_{0}\right)$ from (7) for 3GPP Case 1.

\section{Simulation And Discussion}

In this Section, we use simulations to further study the performance of SCNs and establish the accuracy of our analysis for 3GPP Case 1 studied in Section IV. According to [8] and [9], we use the following parameters: $d_{1}=0.3 \mathrm{~km}$, $\alpha^{\mathrm{L}}=2.09, \alpha^{\mathrm{NL}}=3.75, A^{\mathrm{L}}=10^{-4.11}, A^{\mathrm{NL}}=10^{-3.29}$, $P=24 \mathrm{dBm}, N_{0}=-95 \mathrm{dBm}$.

\section{A. Validation and Discussion of $p^{\text {cov }}(\lambda, \gamma)$}

The results of $p^{\text {cov }}(\lambda, \gamma)$ with $\gamma=1$ and $\gamma=10$ are plotted in Fig. 1. As can be observed from both figures, our analytical results perfectly match the simulation results. Since the results of $A^{\mathrm{ASE}}\left(\lambda, \gamma_{0}\right)$ are computed based on $p^{\mathrm{cov}}(\lambda, \gamma)$, we will only use analytical results for $p^{\mathrm{cov}}(\lambda, \gamma)$ in our discussion hereafter. For comparison, we have also included analytical results assuming a simplistic path loss model that does not differentiate LoS and NLoS transmissions [2]. Note that in [2], only one path loss exponent is defined and denoted by $\alpha$. Here, $\alpha$ is set to $\alpha^{\mathrm{NL}}$ to show the analytical results from [2]. 


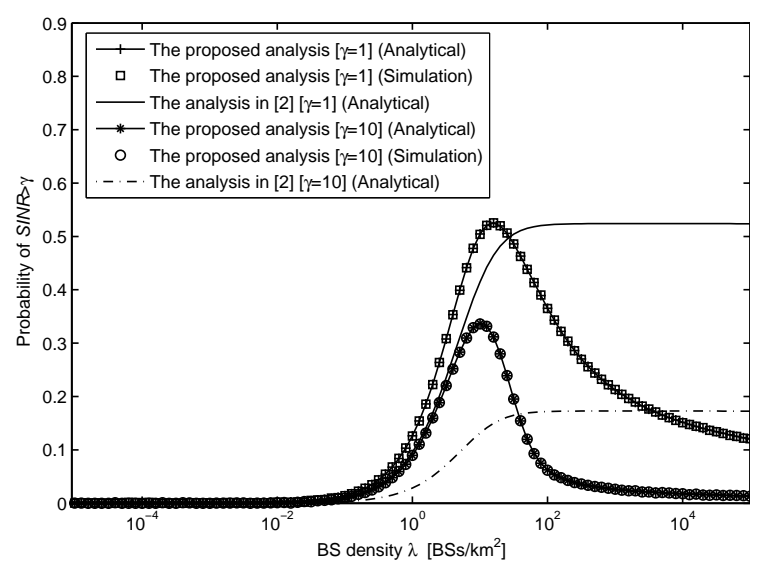

Fig. 1. $p^{\operatorname{cov}}(\lambda, \gamma)$ vs $\lambda$ for 3GPP Case 1 .

From Fig. 1, we can observe that the coverage probability performance given by the stochastic geometry analysis in [2] first increases with the BS density because more BSs provide better coverage in noise-limited networks. Then, when $\lambda$ is large enough, the coverage probability becomes independent of $\lambda$ since the network is pushed into the interference-limited region, e.g., $\lambda>10^{2} \mathrm{BSs} / \mathrm{km}^{2}$ for the analysis in [2]. This observation is consistent with the conclusion in [2], which shows that for a sufficiently large $\lambda$, the coverage probability becomes almost a constant with the increase of the small cell density. The intuition behind the observation is that with the simplistic assumption on the path loss model, the increase in interference power is counterbalanced by the increase in signal power in a interference-limited network, and thus the coverage probability remains the same as $\lambda$ increases.

In Fig. 1, the coverage probability performance of the proposed stochastic geometry analysis incorporating both LoS and NLoS transmissions exhibits a significant deviation from that of the analysis from [2], because when the distance $r$ decreases, or equivalently when the small cell density $\lambda$ increases, LoS transmission occurs with an increasingly higher probability than NLoS transmission. Specifically, when the $\mathrm{SCN}$ is sparse and thus noise-limited, e.g., $\lambda \leq 10 \mathrm{BSs} / \mathrm{km}^{2}$, the coverage probability given by the proposed analysis grows as $\lambda$ increases for the same reason as explained in the above paragraph, i.e., deploying more small cells is beneficial for removing coverage holes. Then, when the network is dense enough and all coverage holes are removed, the coverage probability given by the proposed analysis decreases as $\lambda$ increases, due to the transition of a large number of interference paths from NLoS to LoS. It is important to note that the coverage probability performance of the proposed analysis for 3GPP Case 1 peaks at a certain value $\lambda_{0}$. When $\lambda$ increases above $\lambda_{0}$, interfering BSs may be very close to the typical $\mathrm{UE}$ and hence their signals may reach the UE via strong LoS paths. Such critical point of $\lambda_{0}$ can be readily obtained by setting the partial derivative of $p^{\text {cov }}(\lambda, \gamma)$ with regard to $\lambda$ to zero, i.e., $\lambda_{0}=\underset{\lambda}{\arg }\left\{\frac{\partial p^{\mathrm{cov}}(\lambda, \gamma)}{\partial \lambda}=0\right\}$. The solution to this equation can be numerically found using a standard bisection searching [11]. In Fig. 1, the numerical results of $\lambda_{0}$ are

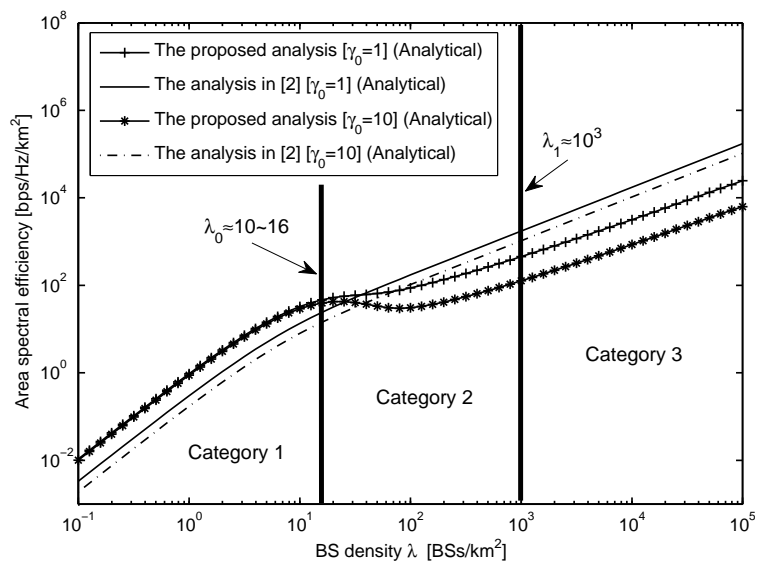

Fig. 2. $A^{\mathrm{ASE}}\left(\lambda, \gamma_{0}\right)$ vs $\lambda$ for $3 \mathrm{GPP}$ Case 1 .

15.85 BSs $/ \mathrm{km}^{2}$ and 10.21 BSs $/ \mathrm{km}^{2}$ for $\gamma=1$ and $\gamma=10$, respectively.

\section{B. Discussion of the Analytical Results of $A^{A S E}\left(\lambda, \gamma_{0}\right)$}

The results of $A^{\mathrm{ASE}}(\lambda, 1)$ and $A^{\mathrm{ASE}}(\lambda, 10)$ are plotted in Fig. 2, comparing the proposed stochastic geometry analysis with the conventional stochastic geometry analysis in [2].

As can be seen from Fig. 2, the analysis from [2] indicates that when the SCN is dense enough, e.g., $\lambda \geq 10^{2} \mathrm{BSs} / \mathrm{km}^{2}$, the ASE performance increases almost linearly with $\lambda$, which is logically correct from the conclusion that $p^{\operatorname{cov}}(\lambda, \gamma)$ is invariable with respect to $\lambda$ for a given $\gamma$ in dense SCNs [2].

In contrast, the proposed stochastic geometry analysis reveals a more complicated trend for the ASE performance. Specifically, when the SCN is relatively sparse, e.g., $\lambda \leq \lambda_{0}$, the ASE quickly increases with $\lambda$ because the network is generally noise-limited, thus adding more small cells immensely benefits the ASE. When the SCN is extremely dense, e.g., $\lambda \geq 10^{4} \mathrm{BSs} / \mathrm{km}^{2}$, the ASE exhibits a nearly linear trajectory with regard to $\lambda$ because both the signal power and the interference power are now LoS dominated and thus statistically stable. Note that the pace is lower than in [2]. As for the practical range of $\lambda$, i.e., $\lambda \in\left[\lambda_{0}, 10^{4}\right] \mathrm{BSs} / \mathrm{km}^{2}$, the ASE first exhibits a slowing-down in the rate of growth (when $\gamma=1$ ) or even a notable decrease in its absolute value (when $\gamma=10)$. This is attributed to the fast decrease of the coverage probability at around $\lambda \in\left[\lambda_{0}, \lambda_{1}\right] \mathrm{BSs} / \mathrm{km}^{2}$ as shown in Fig. 1, where $\lambda_{1}$ is another threshold larger than $\lambda_{0}$. When $\lambda \geq \lambda_{1}$, the ASE will pick up the growth rate as the decrease of the coverage probability becomes very gentle. In Fig. 2, the value of $\lambda_{1}$ seems to be around $10^{3} \mathrm{BSs} / \mathrm{km}^{2}$.

Our new finding indicates the significant impact of the path loss model incorporating both NLoS and LoS transmissions. As a confirmation, noting that in Fig. 1, we can observe that increasing $\gamma$ from 1 to 10 will greatly accelerate the decrease of the coverage probability at around $\lambda \in\left[10,10^{2}\right] \mathrm{BSs} / \mathrm{km}^{2}$, which in turn causes the notable decrease of the ASE at that range of $\lambda$ when $\gamma=10$ in Fig. 2.

With the defined thresholds $\lambda_{0}$ and $\lambda_{1}$, SCNs can be roughly classified into 3 categories, i.e., the sparse $\operatorname{SCN}\left(0<\lambda \leq \lambda_{0}\right)$, 
the dense $\operatorname{SCN}\left(\lambda_{0}<\lambda \leq \lambda_{1}\right)$ and the very dense $\operatorname{SCN}(\lambda>$ $\left.\lambda_{1}\right)$. The ASEs for both the sparse SCN and the very dense SCN grow almost linearly with the increase of $\lambda$, while the ASE of the dense SCN shows a slow growth or even a notable decrease with the increase of $\lambda$. From Fig. 2, we can get a new look at the ultra-dense SCN, which has been identified as one of the key enabling technologies of the 5G networks [1]. Up to now, there is no consensus in both industry and academia on that at what density a SCN can be categorized as an ultra-dense $\mathrm{SCN}$. According to our study, for 3GPP Case 1, we propose that the $5 \mathrm{G}$ systems should target the third category of SCNs as ultra-dense SCNs, i.e., the SCNs with $\lambda>\lambda_{1}$, because the associated ASE will grow almost linearly as $\lambda$ increases. Numerically speaking, $\lambda_{1}$ is around $10^{3} \mathrm{BSs} / \mathrm{km}^{2}$ from Fig. 2 . It is particularly important to note that the second category of SCNs $\left(\lambda_{0}<\lambda \leq \lambda_{1}\right)$ is better avoided in practical SCN deployments due to its cost-inefficiency shown in Fig. 2.

\section{CONCLUSION}

In this paper, we have shown that a sophisticated path loss model incorporating both LoS and NLoS transmissions has a significant impact on the performance of SCNs, measured by the two metrics of the coverage probability and the ASE. Such impact is not only quantitative but also qualitative. Specifically, our theoretical analysis have concluded that the ASE will initially increase with the increase of the small cell density, but when the density of small cells is larger than a threshold $\lambda_{0}$, the network coverage probability will decrease, which in turn makes the ASE suffer from a slow growth or even a notable decrease as the small cell density increases. Furthermore, the ASE will grow almost linearly as the small cell density increases above another larger threshold $\lambda_{1}$. According to our study, for 3GPP cases, we propose that the $5 \mathrm{G}$ systems should target the SCNs with $\lambda>\lambda_{1}$ as ultradense SCNs. Numerically speaking, $\lambda_{1}$ appears to be around several $10^{3} \mathrm{BSs} / \mathrm{km}^{2}$.

The intuition behind our conclusion is that when the density of small cells is larger than a threshold, the interference power will increase faster than the signal power due to the transition of a large number of interference paths from NLoS to LoS, and thus the small cell density matters!

As our future work, we will incorporate more sophisticated UE association strategies and more practical multi-path fading model into the analysis of SCNs because the multi-path fading model is also affected by LoS and NLoS transmissions.

\section{Appendix A: Proof of Theorem 1}

From (4) and (5), we can derive $p^{\text {cov }}(\lambda, \gamma)$ straightforwardly as

$$
\begin{aligned}
p^{\mathrm{cov}}(\lambda, \gamma) & \stackrel{(a)}{=} \int_{r>0} \operatorname{Pr}[\operatorname{SINR}>\gamma \mid r] f_{R}(r) d r \\
& =\int_{r>0} \operatorname{Pr}\left[\frac{P \zeta(r) h}{I_{r}+N_{0}}>\gamma \mid r\right] f_{R}(r) d r \\
& \triangleq \sum_{n=1}^{N}\left(T_{n}^{\mathrm{L}}+T_{n}^{\mathrm{NL}}\right)
\end{aligned}
$$

where $T_{n}^{\mathrm{L}}$ and $T_{n}^{\mathrm{NL}}$ are piece-wise functions defined as $T_{n}^{\mathrm{L}}=\int_{d_{n-1}}^{d_{n}} \operatorname{Pr}\left[\frac{P \zeta_{n}^{\mathrm{L}}(r) h}{I_{r}+N_{0}}>\gamma\right] f_{R, n}^{\mathrm{L}}(r) d r$ and $T_{n}^{\mathrm{NL}}=$ $\int_{d_{n-1}}^{d_{n}} \operatorname{Pr}\left[\frac{P \zeta_{n}^{\mathrm{NL}}(r) h}{I_{r}+N_{0}}>\gamma\right] f_{R, n}^{\mathrm{NL}}(r) d r$, respectively. Besides, $d_{0}$ and $d_{N}$ are respectively defined as 0 and $\infty$. Moreover, $f_{R, n}^{\mathrm{L}}(r)$ and $f_{R, n}^{\mathrm{NL}}(r)$ are the piece-wise PDFs of the event that the UE is associated with the nearest BS with an LoS path at distance $r$ and the event that the UE is associated with the nearest BS with an NLoS path at distance $r$, respectively.

Regarding $f_{R, n}^{\mathrm{L}}(r)$, we define two events in the following, whose joint event is equivalent to the event that the UE is associated with a BS with an LoS path at distance $r$.

- Event $B$ : the nearest BS is located at distance $r$

- Event $D^{\mathrm{L}}$ : the BS is one with an LoS path

According to [2], the cumulative density function (CDF) of Event $B$ with regard to $r$ is given by

$$
F_{R, n}^{B}(r)=1-\exp \left(-\pi r^{2} \lambda\right), \quad\left(d_{n-1}<r \leq d_{n}\right) .
$$

Hence, taking the derivative of $F_{R, n}^{B}(r)$ with regard to $r$, yields the PDF of Event $B$ as

$$
f_{R, n}^{B}(r)=\exp \left(-\pi r^{2} \lambda\right) \times 2 \pi r \lambda, \quad\left(d_{n-1}<r \leq d_{n}\right) .
$$

The PDF $f_{R, n}^{B}(r)$ should be further thinned by the probability of Event $D^{\mathrm{L}}$ on condition of $r$, which is $\operatorname{Pr}_{n}^{\mathrm{L}}(r)$, so that we can get the PDF of the joint event of $B$ and $C^{\mathrm{NL}}$ as

$$
f_{R, n}^{\mathrm{L}}(r)=\operatorname{Pr}_{n}^{\mathrm{L}}(r) \times f_{R, n}^{B}(r) .
$$

Regarding $f_{R, n}^{\mathrm{NL}}(r)$, we also define two events in the following, whose joint event is equivalent to the event that the $\mathrm{UE}$ is associated with a BS with an NLoS path at distance $r$.

- Event $B$ : the nearest BS is located at distance $r$

- Event $D^{\mathrm{NL}}$ : the BS is one with an NLoS path

Similar to (34), the PDF $f_{R, n}^{B}(r)$ should be further thinned by the probability of Event $D^{\mathrm{L}}$ on condition of $r$, which is $\left(1-\operatorname{Pr}_{n}^{\mathrm{L}}(r)\right)$, so that we can get the PDF of the joint event of $B$ and $D^{\mathrm{L}}$ as

$f_{R, n}^{\mathrm{NL}}(r)=$

$$
\left(1-\operatorname{Pr}_{n}^{\mathrm{L}}(r)\right) \times \exp \left(-\pi r^{2} \lambda\right) \times 2 \pi r \lambda,\left(d_{n-1}<r \leq d_{n}\right) .
$$

As for the calculation of $\operatorname{Pr}\left[\frac{P \zeta_{n}^{\mathrm{L}}(r) h}{I_{r}+N_{0}}>\gamma\right]$ in (31), we have

$$
\begin{aligned}
\operatorname{Pr}\left[\frac{P \zeta_{n}^{\mathrm{L}}(r) h}{I_{r}+N_{0}}>\gamma\right] & =\mathbb{E}_{\left[I_{r}\right]}\left\{\operatorname{Pr}\left[h>\frac{\gamma\left(I_{r}+N_{0}\right)}{P \zeta_{n}^{\mathrm{L}}(r)}\right]\right\} \\
& =\mathbb{E}_{\left[I_{r}\right]}\left\{\bar{F}_{H}\left(\frac{\gamma\left(I_{r}+N_{0}\right)}{P \zeta_{n}^{\mathrm{L}}(r)}\right)\right\},
\end{aligned}
$$

where $\mathbb{E}_{[X]}\{\cdot\}$ denotes the expectation operation by taking the expectation over the variable $X$ and $\bar{F}_{H}(h)$ denotes the complementary cumulative density function (CCDF) of RV $h$. Since we assume $h$ to be an exponential RV, we have $\bar{F}_{H}(h)=$ $\exp (-h)$ and thus (36) can be further derived as

$$
\begin{aligned}
\operatorname{Pr}\left[\frac{P \zeta_{n}^{\mathrm{L}}(r) h}{I_{r}+N_{0}}>\gamma\right] & =\mathbb{E}_{\left[I_{r}\right]}\left\{\exp \left(-\frac{\gamma\left(I_{r}+N_{0}\right)}{P \zeta_{n}^{\mathrm{L}}(r)}\right)\right\} \\
& =\exp \left(-\frac{\gamma N_{0}}{P \zeta_{n}^{\mathrm{L}}(r)}\right) \mathscr{L}_{I_{r}}\left(\frac{\gamma}{P \zeta_{n}^{\mathrm{L}}(r)}\right),
\end{aligned}
$$


where $\mathscr{L}_{I_{r}}(s)$ is the Laplace transform of $I_{r}$ evaluated at $s$.

As for the calculation of $\operatorname{Pr}\left[\frac{P \zeta_{n}^{\mathrm{NL}}(r) h}{I_{r}+N_{0}}>\gamma\right]$ in (31), similar to (36), we have

$\operatorname{Pr}\left[\frac{P \zeta_{n}^{\mathrm{NL}}(r) h}{I_{r}+N_{0}}>\gamma\right]=\exp \left(-\frac{\gamma N_{0}}{P \zeta_{n}^{\mathrm{NL}}(r)}\right) \mathscr{L}_{I_{r}}\left(\frac{\gamma}{P \zeta_{n}^{\mathrm{NL}}(r)}\right)$

Our proof of Theorem 1 is completed by plugging (34), (35), (37), and (38) into (31).

\section{APPENDIX B: PROOF OF LEMMA 2}

Based on Theorem 1, it is straightforward to derive $\mathscr{L}_{I_{r}}(s)$ in the range of $0<r \leq d_{1}$ as

$$
\begin{aligned}
& \mathscr{L}_{I_{r}}(s)=\mathbb{E}_{\left[I_{r}\right]}\left\{\exp \left(-s I_{r}\right) \mid 0<r \leq d_{1}\right\} \\
&=\mathbb{E}_{\left[\Phi,\left\{\beta_{i}\right\},\left\{g_{i}\right\}\right]}\left\{\exp \left(-s \sum_{i \in \Phi / b_{o}} P \beta_{i} g_{i}\right) \mid 0<r \leq d_{1}\right\} \\
&=\mathbb{E}_{[\Phi]}\left\{\prod_{i \in \Phi / b_{o}} \mathbb{E}_{[\beta, g]}\{\exp (-s P \beta g)\} \mid 0<r \leq d_{1}\right\} \\
& \stackrel{(a)}{=} \exp \left(-2 \pi \lambda \int_{r}^{\infty}\left(1-\mathbb{E}_{[g]}\{\exp (-s P \beta(u) g)\}\right) u d u \mid\right. \\
&\left.0<r \leq d_{1}\right),
\end{aligned}
$$

where (a) in (39) is obtained from [2].

Since $0<r \leq d_{1}, \mathbb{E}_{[g]}\{\exp (-s P \beta(u) g)\}$ in (39) should consider interference from both LoS and NLoS paths. Thus, $\mathscr{L}_{I_{r}}(s)$ can be further derived as

$$
\begin{aligned}
\mathscr{L}_{I_{r}}(s)= & \exp \left(-2 \pi \lambda \int_{r}^{d_{1}}\left(1-\frac{u}{d_{1}}\right) \frac{u}{1+\left(s P A^{\mathrm{L}}\right)^{-1} u^{\alpha^{\mathrm{L}}}} d u\right) \\
& \times \exp \left(-2 \pi \lambda \int_{r_{1}}^{d_{1}} \frac{u}{d_{1}} \frac{u}{1+\left(s P A^{\mathrm{NL}}\right)^{-1} u^{\alpha^{\mathrm{NL}}}} d u\right) \\
& \times \exp \left(-2 \pi \lambda \int_{d_{1}}^{\infty} \frac{u}{1+\left(s P A^{\mathrm{NL}}\right)^{-1} u^{\alpha^{\mathrm{NL}}}} d u\right) \cdot(40)
\end{aligned}
$$

Plugging $s=\frac{\gamma r^{\alpha^{\mathrm{L}}}}{P A^{\mathrm{L}}}$ into (40), and considering the definition of $\rho_{1}(\alpha, \beta, t, d)$ and $\rho_{2}(\alpha, \beta, t, d)$ in (20) and (21), we can obtain $\mathscr{L}_{I_{r}}\left(\frac{\gamma r^{\alpha^{\mathrm{L}}}}{P A^{\mathrm{L}}}\right)$ shown in (19), which concludes our proof.

\section{ApPendix C: PROOF OF LEMMA 3}

Following the same approach in Appendix B, it is ready to derive $\mathscr{L}_{I_{r}}\left(\frac{\gamma r^{\alpha^{\mathrm{NL}}}}{P A^{\mathrm{NL}}}\right)$ in the range of $0<r \leq x_{1}$ as $\mathscr{L}_{I_{r}}\left(\frac{\gamma r^{\alpha^{\mathrm{NL}}}}{P A^{\mathrm{NL}}}\right)=$

$$
\begin{aligned}
& \exp \left(-2 \pi \lambda \int_{r_{2}}^{d_{1}}\left(1-\frac{u}{d_{1}}\right) \frac{u}{1+\left(\frac{\gamma r^{\mathrm{NL}}}{P A^{\mathrm{NL}}} P A^{\mathrm{L}}\right)^{-1} u^{\alpha^{\mathrm{L}}}} d u\right) \\
& \times \exp \left(-2 \pi \lambda \int_{r}^{d_{1}} \frac{u}{d_{1}} \frac{u}{1+\left(\frac{\gamma r^{\alpha^{\mathrm{NL}}}}{P A^{\mathrm{NL}}} P A^{\mathrm{NL}}\right)^{-1} u^{\alpha^{\mathrm{NL}}}} d u\right) \\
& \times \exp \left(-2 \pi \lambda \int_{d_{1}}^{\infty} \frac{u}{1+\left(\frac{\gamma r^{\mathrm{NL}}}{P A^{\mathrm{NL}}} P A^{\mathrm{NL}}\right)^{-1} u^{\alpha^{\mathrm{NL}}}} d u\right) \cdot
\end{aligned}
$$

Similarly, $\mathscr{L}_{I_{r}}\left(\frac{\gamma r^{\alpha^{\mathrm{NL}}}}{P A^{\mathrm{NL}}}\right)$ in the range of $x_{1}<r \leq d_{1}$ can be calculated by

$$
\begin{aligned}
& \mathscr{L}_{I_{r}}\left(\frac{\gamma r^{\alpha^{\mathrm{NL}}}}{P A^{\mathrm{NL}}}\right)= \\
& \quad \exp \left(-2 \pi \lambda \int_{r}^{d_{1}} \frac{u}{d_{1}} \frac{u}{1+\left(\frac{\gamma r^{\mathrm{NL}}}{P A^{\mathrm{NL}}} P A^{\mathrm{NL}}\right)^{-1} u^{\alpha^{\mathrm{NL}}}} d u\right) \\
& \quad \times \exp \left(-2 \pi \lambda \int_{d_{1}}^{\infty} \frac{u}{1+\left(\frac{\gamma r^{\mathrm{NL}}}{P A^{\mathrm{NL}}} P A^{\mathrm{NL}}\right)^{-1} u^{\alpha^{\mathrm{NL}}}} d u\right) .
\end{aligned}
$$

Our proof is thus completed by plugging (20) and (21) into (41) and (42).

\section{APPENDiX D: ProOF OF LEMMA 4}

Following the same approach in Appendix B, it is ready to derive $\mathscr{L}_{I_{r}}\left(\frac{\gamma r^{\alpha^{\mathrm{NL}}}}{P A^{\mathrm{NL}}}\right)$ in the range of $r>d_{1}$ as

$$
\begin{aligned}
\mathscr{L}_{I_{r}}\left(\frac{\gamma r^{\alpha^{\mathrm{NL}}}}{P A^{\mathrm{NL}}}\right) & =\exp \left(-2 \pi \lambda \int_{r}^{\infty} \frac{u}{1+\left(\gamma r^{\alpha^{\mathrm{NL}}}\right)^{-1} u^{\alpha^{\mathrm{NL}}}} d u\right) \\
& =\exp \left(-2 \pi \lambda \rho_{2}\left(\alpha^{\mathrm{NL}}, 1,\left(\gamma r^{\alpha^{\mathrm{NL}}}\right)^{-1}, r\right)\right),
\end{aligned}
$$

where $\rho_{2}(\alpha, \beta, t, d)$ is defined in (21).

Our proof is thus completed with (43).

\section{REFERENCES}

[1] D. López-Pérez, M. Ding, H. Claussen, and A. H. Jafari, "Towards $1 \mathrm{Gbps} / \mathrm{UE}$ in cellular systems: understanding ultra-Dense small cell deployments," arXiv:1503.03912 [cs.NI], Mar. 2015.

[2] J. G. Andrews, F. Baccelli, and R. K. Ganti, "A tractable approach to coverage and rate in cellular networks," IEEE Trans. Commun., vol. 59, no. 11 , pp. 3122-3134, Nov. 2011.

[3] M. Haenggi, J. G. Andrews, F. Baccelli, O. Dousse, and M Franceschetti, "Stochastic geometry and random graphs for the analysis and design of wireless networks," IEEE J. Sel. Areas Commun., vol. 27, no. 7, pp. 1029-1046, Sep. 2009.

[4] H. Dhillon, R. Ganti, F. Baccelli, and J. Andrews, "Modeling and analysis of K-tier downlink heterogeneous cellular networks," IEEE J. Sel. Areas Commun., vol. 30, no. 3, pp. 550-560, Apr. 2012

[5] S. Singh, H. S. Dhillon, and J. G. Andrews, "Offloading in heterogeneous networks: Modeling, analysis, and design insights," IEEE Trans. Wireless Commun., vol. 12, no. 5, pp. 2484-2497, Apr. 2013.

[6] X. Zhang and J. G. Andrews, "Downlink cellular network analysis with multi-slope path loss models," arXiv:1408.0549 [cs.NI], 2014.

[7] T. Bai and R. W. Heath Jr., "Coverage and rate analysis for millimeter wave cellular networks," arXiv:1402.6430 [cs.NI], 2014.

[8] 3GPP, "TR 36.828 (V11.0.0): Further enhancements to LTE Time Division Duplex (TDD) for Downlink-Uplink (DL-UL) interference management and traffic adaptation," Jun. 2012.

[9] Spatial Channel Model AHG (Combined ad-hoc from 3GPP \& 3GPP2), "Spatial Channel Model Text Description,"Apr. 2003.

[10] I.S. Gradshteyn and I.M. Ryzhik, Table of Integrals, Series, and Products (7th Ed.), Academic Press, 2007.

[11] R. L. Burden and J. D. Faires, Numerical Analysis (3rd Ed.), PWS Publishers, 1985. 